\title{
Homo duplex
}

Par François Azouvi (C. N. R.S.)

En 1805, dans le Mémoire sur la décomposition de la pensée, Maine de Biran expose pour la première fois sa doctrine définitive concernant la génèse du sujet. Dans ce processus, le philosophe tient à distinguer deux étapes qui correspondent en profondeur à deux strates de l'être humain: au commencement sont les «affections pures», la part passive de l'être vivant et sentant, encore incapable de savoir qu'il vit et qu'il sent; ces purs affects sont le résultat des fonctions organiques qui «conspirent» dans une vie infraconsciente. L'homme proprement dit ne surgit qu'au moment où il peut dire je, grâce à l'exercice de la motricité volontaire. Dans la première étape, et toutes les fois que les affections prennent le pas sur la libre initiative du sujet, nous avons à faire à l'«être simple dans la vitalité». Avec les déterminations réfléchies de l'être conscient, nous découvrons «l'homme double dans l'humanité». ${ }^{1}$ Biran ne fait-il là que restaurer le dualisme traditionnel du corps et de l'âme? Justement pas; la division à laquelle il procède, et qui partage le sujet en deux instances irréductibles mais inséparables, ne doit rien à Descartes: ce sont deux fonctions qu'il distingue, et non deux substances. Aussi les maîtres dont il se réclame ne sont-ils pas des philosophes mais un médecin et un naturaliste: c'est dans les Praelectiones de morbis nervorum de Boerhaave que Maine de Biran a lu la formule: «homo duplex in humanitate, simplex in vitalitate ${ }^{2}$; et c'est dans l'Histoire naturelle de Buffon qu'il a trouvé un long développement intitulé: Homo duplex ${ }^{3}$.

L'usage philosphique que Biran fait de la formule: homo duplex, est rendu possible par le sens nouveau que lui confèrent, au $\mathrm{XVIII}^{\mathrm{e}}$ siècle, anthropologues et physiologistes. L'expression a pourtant un long passé; c'est en reprenant une vieille formule, forgée dans un contexte absolument différent, mais en lui conférant une signification originale, que Boerhaave et quelques autres ont contribué à la rendre de nouveau féconde.

D'où vient-elle? C'est dans un sens moral qu'elle est employée d'abord, du moins à ma connaissance. L'homme double, et même l'homme multiple, chez Platon, désignent celui qui, au lieu d'être cordonnier, marin ou cultivateur, pratique deux ou trois de ces fonctions. C'est un homme «mélangé», à qui plaira un art mixte, symptôme d'une impureté préjudiciable à son bien 
propre et à celui de la collectivité ${ }^{4}$. Les Stö̈ciens reprendront cette idée d'un homme divisé intérieurement, et leur sagesse s'exprimera à cet égard dans les mêmes termes que celle de Platon: «Tu dois être un seul homme.» ${ }^{5}$

Un seul homme: pour des raisons proches, c'est aussi la recommandation que nous lisons dans les Psaumes et dans l'Ecclésiastique: une «langue double», un «cœur double», ce sont les signes de la fausseté et de la crainte du Seigneur ${ }^{6}$. Dans le prolongement de cette condamnation l'Epître de Jacques

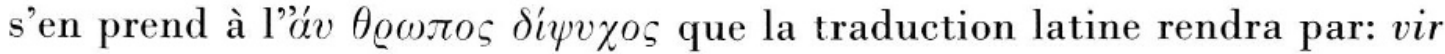
duplex animo: «... le tergiverseur ressemble au flot de la mer qu'agite et repousse le vent. Que cet homme-là ne présume pas recevoir quoi que ce soit du Seigneur. C'est un homme irrésolu, inconstant dans tous ses chemins.» ${ }^{7}$

Parce qu'elle nuit à l'harmonie (individuelle et sociale), ou parce qu'elle détourne de Dieu, la dualité intérieure est donc d'abord condamnée comme une sorte de duplicité. Le rusé Ulysse, à en croire Horace, est duplex (Odes, I, 67). Mais de la ruse au double jeu, il n'y a qu'un pas, et l'homo duplex sera synonyme d'homo bilinguis, fictus, simulatus $^{8}$.

Mais un homme capable de duplicité, n'est-ce pas un homme déchiré, divisé en deux natures inconciliables? La dualité morale implique une dualité plus fondamentale: l'homme est double, corps et âme, terre et ciel, mortel et immortel.

Platon a fourni tous les éléments d'un tel dualisme, ainsi que la raison, constamment invoquée après lui, qui y préside: c'est parce que l'homme connaît le conflit, c'est parce qu'il est capable de désirer ce qu'il ne veut pas, qu'on doit conclure qu'il n'est pas un être simple. La division s'atteste de la souffrance. Platon cite à cette fin le cas de l'hydropique qui désire et ne veut pas boire: comment une telle sécession intérieure serait-elle possible s'il n'y avait en l'homme deux parts, l'une rationnelle, l'autre irrationnelle et désirante ${ }^{9}$ ? Pour des usages bien différents, on trouve le même argument déjà dans le traité hippocratique De natura hominis - où il sert à établir que l'homme n'est pas simple, mais constitué de quatre éléments ${ }^{10}$-, et plus tard dans l'anthropologie paulinienne, où il fonde la distinction entre l'homme intérieur et l'homme extérieur, entre la loi et l'esprit de la loi des membres ${ }^{11}$. «Il y a deux hommes en chacun de nous», commente Origène, et si l'homme intérieur a été créé à l'image de Dieu, on peut dire également que l'homme extérieur et l'homme intérieur se correspondent terme à terme: l'homme extérieur a des membres, des yeux, des oreilles, etc.; l'homme intérieur aussi, mais il faut l'entendre en un autre sens, «spiritualiter» dira saint Augustin ${ }^{12}$.

Dès les premiers siècles, on voit que sont solidement établies l'idée d'une 
opposition entre les parts immortelle et mortelle de l'homme, en même temps que celle d'une correspondance: l'homme extérieur est l'enveloppe de l'homme intérieur, mais cette enveloppe n'est pas sans convenir à ce qu'elle contient. L'une est l'image de l'autre: nous verrons la métaphore resurgir, dans une perspective tout à fait différente, chez des médecins du XVII ${ }^{\mathrm{e}}$ et du XVIII ${ }^{\mathrm{e}}$ siècles.

Appliquée à l'homme pour exprimer sa division en deux parts, immortelle et mortelle, la formule homme double apparaît littéralement dans le Poimandrès et dans l'Asclepius, qui font partie des écrits attribués à Hermès Trismégiste. La date de leur composition est imprécise, mais se situe très vraisemblablement au second ou au troisième siècle. Ils recueillent et mêlent des éléments platoniciens christianisés et surtout des thèmes gnostiques.

Selon le Poimandrès, l'homme est le fruit des amours de l'Homme

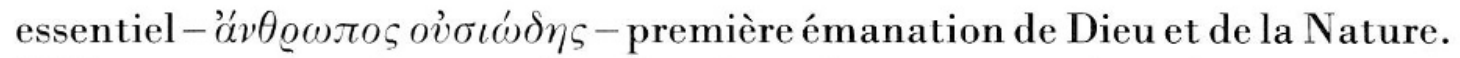
L'Homme essentiel, entré dans la sphère démiurgique, brise les armatures des sept cercles de l'Univers, se penche dans l'ouverture et montre «à la Nature d'en-bas la belle forme de Dieu». Celle-ci se reflète en effet dans l'eau, et c'est de ce reflet que s'éprend la Nature, mais aussi l'Homme essentiel qui contemple sa propre image, l'aime et veut habiter là où il la voit. C'est ainsi qu'il descend dans «la forme sans raison» et s'unit à elle. «Et c'est pourquoi, seul de tous les êtres qui vivent sur la terre, l'homme est double, mortel de par le corps, immortel de par l'Homme essentiel.» ${ }^{13} L^{\prime}$ Asclepius précise en latin: la partie que «les Grecs nomment essentielle et nous, formée à la ressemblance de Dieu», est simple tandisqu'est quadruple le corps constitué à partir des quatre éléments ${ }^{14}$.

Il semble que ce soit au quatrième siècle que l'expression: homme double entre dans la littérature chrétienne. Dans le cadre des disputes relatives à la nature du Christ, avant le Concile de Nicée, la formule: homme double, est bien venue, même si elle est contestable, pour dire que Jésus est à la fois homme et Dieu. Aussi ne s'étonne-t-on pas de la trouver chez Grégoire de Nazianze, qui a connu certains des textes hermétiques: «[le Christ] a été certes envoyé, mais comme un homme, car il est double». ${ }^{15}$ Sans doute faut-il veiller à ne pas faire disparaître l'unité de la personne qu'est Jésus derrière la dualité de sa nature: Nestorius, à cette fin, rappelle qu'il n'y a pas deux Christ, mais un seul, et oppose la dualité en nature et la dualité en dignité ${ }^{16}$.

Il n'en va pas autrement, toutes proportions gardées, de l'être humain luimême: la composition de l'âme et du corps, en l'homme, est convenablement exprimée par une comparaison tirée de la double nature du Christ. Ici encore, 
la formule est risquée mais tentante: Cyrille d'Alexandrie refuse de l'employer, dans un cas comme dans l'autre, pour insister sur le fait que nous distinguons par la seule pensée les deux natures, tout en ne percevant qu'un seul homme, qu'un seul vivant ${ }^{17}$. Au moins ne récuse-t-il pas l'analogie entre l'homme et l'Homme-Dieu. D'autres étaient moins prudents et n'hésitaient pas à parler d'un «homme double», semblable à Dieu par son âme, aux bêtes par son corps. A cet homme double convient une double purification: celle de l'eau pour le corps, celle de l'Esprit saint pour l'âme ${ }^{18}$. Aussi marquée, par conséquent, que soit l'opposition entre le corps et l'âme, entre les parts mortelle et immortelle, on voit que demeure entre elles une analogie qui autorise l'emploi d'une même image.

La vie est donc double: les auteurs chrétiens s'accordent sur ce point avec les platoniciens comme avec les gnostiques, Jamblique avec Grégoire de Nysse: qu'elle soit rapportée à Platon ou expliquée par le péché d'Adam, l'idée selon laquelle l'homme vit d'une double vie s'impose généralement ${ }^{19}$. L'une est une vie immortelle, céleste; c'est une vraie vie. L'autre est une vie terrestre, corporelle; c'est presque une mort. Saint Bernard au onzième siècle, Ruysbroeck au quatorzième, opposeront la «vie vivante» et la «vie mortelle». ${ }^{20}$

Vivre a deux significations, et l'une d'elles est chargée d'une valeur dont l'autre est dépourvue. Entre les deux parts de l'homme double, une proportion règne et une correspondance peut être instaurée. Mais il y a pourtant entre elles l'écart qu'il y a entre le ciel et la terre. Car la dualité de l'homme est le résultat d'une chute, d'une «audace» pour parler comme Plotin. Qu'on se souvienne du récit du Poimandrès: l'Homme, trop curieux de ce qui se passe au-dessous des sept cercles célestes, s'éprend de sa propre image dans le miroir que lui tend la Nature; ce qu'il aime en elle, c'est encore lui. Et l'homme est le résultat de ce coupable amour de soi.

Mais la part immortelle de l'homme est ici une part vraiment divine, et l'être en qui elle réside est, selon une formule célèbre de l'Asclepius, «un grand miracle». L'homme n'est pas seulement, comme dans la Genèse, à l'image de Dieu, il est lui aussi un dieu même s'il est déchu. D'ailleurs, il façonne et anime des statues à l'effigie divine, et cela seul atteste de sa nature et de son origine.

Lorsque les renaissants, à la suite de Marsile Ficin qui obéissait lui-même à la demande pressante de Côme de Médicis, découvrent, éditent et traduisent, un peu partout en Europe, les écrits hermétiques, ils y découvrent une sagesse qu'ils croient antérieure à tout, mais où ils ne tardent pas à voir 
une anthropologie bien conforme à leurs propres vues. Plus que sur l'idée de chute, c'est sur le thème de l'homme divin qu'ils vont insister, et pour cela, «l'homme double» viendra à point nommé ${ }^{21}$.

Souvenons-nous des mots par lesquels débute le discours que Pic de la Mirandole intitule: De la dignité de l'homme: «Vénèrables Pères! J'ai lu dans les livres anciens des arabes que le sarrasin Abdallah, à qui l'on demandait quel était, à ses yeux, le spectacle le plus admirable sur cette sorte de scène qu'est le monde, avait répondu qu'il n'y voyait rien de plus merveilleux que l'homme. Cette opinion concorde avec celle de Mercure: ‘Asclepius, l'homme c'est un grand miracle!»» ${ }^{22}$ Nous connaissons la nature de ce miracle: c'est que l'homme ne soit ni mortel, ni immortel, ni céleste, ni terrestre. Sa «double nature» ${ }^{23}$ fait qu'il est les deux, qu'aucune place fixe ne lui est assignée dans la Création, qu'il peut librement se porter vers les choses célestes ou se précipiter vers les infernales. Marsile dit volontiers ${ }^{24}$ - en mêlant aux textes hermétiques ses souvenirs d'Avicenne ${ }^{25}$ - que l'âme est un véritable Janus: comme le dieu romain, elle a un double visage qui lui permet de regarder à la fois vers le haut et vers le bas.

Chez Marsile comme chez Pic, la dualité de l'homme est maintenant sa chance, son risque: parce qu'il est double, l'homme est un être intermédiaire dont la vie, pour reprendre une expression de Ficin, «touche aux deux vies extrêmes». ${ }^{26}$ Il est le lien de la terre et du ciel, la copula mundi; sans être radicalement neuf ${ }^{27}$, le thème subit de la part des renaissants une exploitation massive, encore accrue par la teneur magique que prend l'idée d'un mariage du ciel et de la terre ${ }^{28}$.

Choisissons deux exemples de la façon renouvelée dont les renaissants interprètent l'homo duplex attribué à Hermès «trois fois trés grand». Le premier est emprunté au commentaire que François de Candale ajoute en 1579 à sa traduction du Poimandrès. Après avoir, en passant, identifié la part immortelle de l'écrit hermétique et l'homme intérieur selon saint Paul, François de Candale insiste sur ce qu'il y a de spécifique en l'homme. Or ce n'est pas douteux: «Entre tous animaux mortels, le seul homme est exempté, de sa nature immortelle, de la puissance de fatum, ou nécessité fatale, à cause qu'il est, en cette partie intelligible, incorporel et du tout hors la sujétion des sens.» ${ }^{29}$ Or le texte original n'en disait pas tant, et disait parfois le contraire. Car, si l'homme est immortel par son origine, il subit néanmoins la condition des mortels et demeure soumis à la Destinée: entre autres signes de cet asservissement, il faut compter le fait d'être désormais mâle ou femelle, une fois passée une brève période d'androgynat ${ }^{30}$. 
Mais c'est peut-être dans le De sapiente de Charles de Bovelles que l'on trouve la plus puissante des réinterprétations que les renaissants font de l'homo duplex ${ }^{31}$. L'homme naturel, selon Bovelles, est un homme déchu, qui «mène une vie indigne de l'homme». C'est la sagesse qui fait de lui un homme complet, achevé, homme selon la nature et selon l'intelligence, homme en puissance et homme en acte, homme en son principe et en sa fin, en réalité et en apparence ${ }^{32}$. Autrement dit, l'homme naturel est un, tandis que le sage est deux: c'est avec raison qu'on le représente sous les traits de Janus bifrons, car il est vraiment double: «le sage n'est pas un homme pur et simple, mais il est deux fois homme (homo geminus) » ${ }^{33}$, formé de l'union de «l'homme de la nature» et de «l'homme substantiel» (homo substantialis). Dans l'homme

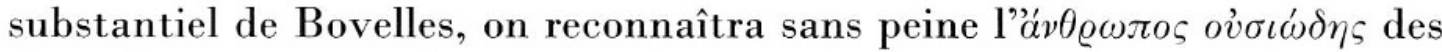
écrits hermétiques. «En raison de cette disposition, celui que la nature n'avait fait qu'homme est appelé, par la grâce et le généreux bienfait de l'art, deux fois homme (reduplicatus homo), c'est-à-dire homme de l'homme». ${ }^{34}$

L'homo duplex n'est donc pas tant le résultat d'une chute que le terme d'une promotion; l'homo simplex est ici un manque, une carence, par rapport aux réalisations de l'homo duplex. Cassirer commente ainsi cette nouvelle définition de la sagesse: «Le sage est celui qui a pris la mesure des contraires qui sont impliqués dans l'essence de l'homme, qui les a reconnus et par làmême surmontés.» ${ }^{35}$ D'où il résulte que l'humanitas est un processus et non un état. Homo duplex: il s'agit pour l'homme de le devenir. Il est à peine besoin d'insister sur l'importance de la réévaluation dont la formule «l'homme est double» est ici l'objet.

C'est à cette époque que l'expression: homme double pénètre chez les médecins et contribue à féconder leur philosophie médicale. Marsile lui-même, auteur d'une De triplici vita, n'était-il pas médecin? mais aussi Symphorien Champier, qui édite et commente en 1507 les textes hermétiques dans son Liber de quadruplici vita; et Agrippa de Nettesheim également, qui leur consacre en 1515 un enseignement au Gymnase de Pavie ${ }^{36}$.

Mais c'est assurément chez Paracelse que l'homme double va être appelé à un rôle considérable. Disons-le d'un mot: l'homme double des écrits hermétiques viendra remplacer l'homme quadruple de la tradition hippocraticogalénique. Paracelse, on le sait, n'a pas de mots assez durs pour fustiger les partisans du système des quatre humeurs, qui ont fourvoyé la médecine. Aussi son ambition n'est-elle rien moins que de la fonder à nouveaux frais. Or Paracelse estime ne pouvoir le faire qu'au prix de ce qu'il appelle lui-même 
un «renversement»: jusqu'à présent les médecins ont tiré leur prétendue science de la considération exclusive de l'homme. Or une telle connaissance est nécessairement fautive car elle est incomplète: «Puisque le médecin doit soigner tous les hommes, comment peut-il le faire s'il n'en connaît qu'un, dix ou cent?» $\mathrm{D}$ 'où cette conséquence: «On n'a écrit que des bribes sans fondement. C'est le monde qui doit done livrer l'homme.» ${ }^{37}$

Le «renversement» auquel Paracelse attache sa doctrine tient donc dans la certitude où il est qu'il n'y a de science médicale que du Tout. «Le médecin doit naître du monde extérieur, et non de l'homme. Si le médecin trouve en l'homme une maladie, pour en connaître le nom et les aspects, il doit [...] s'instruire, expérimenter au-dehors. Impossible de reconnaître la maladie en examinant le seul foyer où elle se développe» ${ }^{38}$. L'impératif catégorique du médecin se résume alors en cette recommandation: «Apprends de même à regarder l'homme à travers la nature.» ${ }^{39}$

On voit bien ce que suppose une telle doctrine: la vieille idée de l'hommemicrocosme. Car s'il est vrai qu'il faut chercher à l'extérieur la connaissance de ce qui se passe à l'intérieur de l'homme, c'est que l'extérieur et l'intérieur se correspondent, que l'homme et le monde sont dans un rapport d'équivalence. L'homme est le «petit monde», l'Univers est le «grand monde», et leur correspondance est rigoureuse, terme à terme. «La compréhension et la connaissance des corps dans la sphère supérieure, d'une part, et dans le microcosme inférieur, d'autre part, correspondent: il s'agit d'un même firmament, d'un même astre, d'une nature et d'une essence unique dans une forme et un aspect dédoublés.» ${ }^{40}$

Mais l'homme en lui-même n'est pas moins double: la division du macrocosme et du microcosme passe en lui, sous la forme d'un homme extérieur et visible, et d'un homme intérieur, invisible: «il existe deux hommes en un seul corps», lit-on dans le De causis morborum invisibilium ${ }^{41}$, ou encore, dans l'Erklärung der ganzen Astronomie: «l'homme en lui-même est également double (zweifach), c'est-à-dire divisé en deux, à savoir en deux corps $[\ldots]$, donc en un corps visible et saisissable et un corps invisible et insaisissable, le premier étant le corps des trois premiers éléments (sel, soufre, mercure) et le second celui des astres». ${ }^{42}$ Car on peut dire indifféremment que l'homme intérieur est «esprit» (par opposition au «sang et à la chair»), ou qu'il est «corps astral». Sans doute cette dualité n'empêche-t-elle pas l'homme d'être un, car l'esprit ne se sépare pas du corps, ni le corps de l'esprit. Dans un manuscrit inédit, Paracelse a cette formule qui ne contredit qu'en apparence les précédentes que nous citions: «seul existe l'homme, et il 
comprend les deux. Ainsi l'homme est un et non deux».4 ${ }^{43}$ Car il reste que cette unité est composée de deux moitiés, dont seule la réunion permet de construire une médecine digne de ce nom. "Les fièvres sont dans les tremblements de terre, etc. Le médecin apprend toutes les maladies à l'extérieur de l'homme.» Prêtons attention à la formule qui suit, car nous la retrouverons chez Boerhaave lorsqu'à son tour il reprendra l'expression: homo duplex: «L'image de l'homme tout entier se forme ainsi en lui. Alors il peut pénètrer à l'intérieur de l'homme. Alors il devient un médecin qui observe les urines, qui tâte le pouls, qui juge de la santé parce qu'il connaît l'homme extérieur, l'homme qui est à la fois le ciel et la terre.» ${ }^{44}$

Le médecin est donc, comme le sage selon Bovelles, un homme double, «divisé en deux sphères, la sphère philosophique et la sphère astronomique». ${ }^{45}$ Science du Tout, c'est la médecine, chez Paracelse, qui surmonte les contradictions de la connaissance morcelée et se forme une «image de l'homme tout entier». N'oublions pas qu'il y a deux sagesses, l'éternelle et la mortelle, puisqu'il y a deux parts en l'homme: aucune des deux ne doit être sacrifiée, car «l'homme doit vivre en deux lumières sans qu'aucune n'entrave l'autre: elles sont conjointes comme époux et épouse». ${ }^{46}$

Exprimée dans l'image de la gémellité chez Bovelles (homo geminus), ou dans celle du mariage chez Paracelse, c'est la même idée d'un homme qui n'est complet que par la réunion de ses deux parts. L'humanité ne réside pas, en toute rigueur, dans le seul homme intérieur ou «essentiel», mais bien dans «l'image de l'homme tout entier», c'est-à-dire dans la synthèse de l'homme intérieur et de l'homme extérieur. Forgée pour rendre compte du manque, de l'incomplétude et des déchirements dont l'homme est la proie, l'expression homme double est maintenant associée au fait de rassembler, de compléter, d'apaiser et de réconcilier. Sa signification se trouve inversée, de même que l'homme, qui est le «petit monde», devient le «grand monde» lorsqu'il accède à la sagesse ${ }^{47}$.

Chez Paracelse, ce thème de l'homme intégral est exprimé dans le langage hermétiste qui est celui de la tradition à laquelle il appartient - tradition que l'on retrouvera dans la théosophie d'un Valentin Weigel ou d'un Jacob Boehme. Franchissons maintenant deux siècles: lorsque Boerhaave, dans ses cours sur les maladies du système nerveux, reprend la formule: homo duplex, il n'entend nullement redonner vie aux spéculations magico-alchimiques de son prédécesseur; il tient même - nous allons le voir - à montrer que les deux hommes qu'il y a en nous sont également «visibles», également corporels. Mais, de façon singulière, Boerhaave et quelques autres après lui, vont 
retrouver quelque chose de l'ancienne notion d'un «homme essentiel», qui, à lui seul de nouveau, constitue «l'image de l'homme tout entier».

Ce n'est donc pas pour redire la division de l'homme en corps et âme que Boerhaave emploie la formule homo duplex. L'auteur des Institutiones medicae professe à cet égard un cartésianisme prudent: tout ce qui a rapport à la pensée doit être attribué à l'esprit seul, tout ce qui concerne l'étendue, la figure et le mouvement, doit être rapporté au corps seul ${ }^{48}$. Le dualisme de Descartes (ou parfois celui de Malebranche) est la toile de fond des analyses du physiologiste.

Mais «l'humanité», ce n'est ni le corps tout seul, ni l'esprit séparé, c'est «l'homme total», «en même temps corps et esprit». ${ }^{49}$ C'est dans le sensorium commune que réside le centre de cette «humanité», définie par l'étendue d'un pouvoir et la réversibilité de ce que l'on appellera bientôt le physique et le moral: «l'humanité, c'est-à-dire le changement des pensées en vue du changement du corps, et inversement, la possibilité de changer le corps par l'intermédiaire du changement de la pensée». ${ }^{50}$ Le sensorium est ce foyer où se croisent et s'échangent sans cesse des sensations et des incitations motrices, transmises les unes et les autres par les nerfs qui s'y rejoignent ${ }^{51}$. Aux parties sensibles, correspondent des filets nerveux issus du sensorium. Mais il y a aussi des organes, pourtant innervés, qui ne sont le siège d'aucune sensation propre: c'est le cas, notamment, du foie et du cœur. En revanche l'inflammation de leurs «membranes» produit une vive douleur. Boerhaave est ainsi conduit à ébaucher une division des systèmes nerveux selon qu'ils président ou non à la sensibilité consciente: «Nous sommes ainsi parvenus à distinguer progressivement les nerfs; nous avons trouvé que des nerfs, servant à la sensation et au mouvement, sont issus du cerveau; et que d'autres, ne servant à rien de tel, ont leur origine dans le cervelet. Supposons que l'on conserve tous les points sensibles du corps humain, mais que l'on ôte tous ceux qui sont insensibles; le cœur serait alors supprimé, et tout ce qui dépend du cœur sans contribuer à la sensation ni au mouvement volontaire; rien dans le foie ne subsisterait, si ce n'est la membrane sensible; en somme, on aurait alors une image de l'homme tout entier, sans les liquides ni les parties insensibles. Donc l'homme est double dans l'humanité, simple dans la vitalité.» ${ }^{52}$

On aura sûrement noté l'expression: «image de l'homme tout entier». On voit que c'est le système nerveux central qui constitue une telle image, véritable abrégé de l'homme intégral. Et nous ne serons pas surpris de trouver, aussitôt après, une allusion à la notion d'homme intérieur, rapportée 
à un bref passage de Sydenham, où celui-ci opposait l'homme extérieur, formé de l'assemblage des parties visibles, et l'homme intérieur, constitué par l'enchaînement des esprit animaux, invisibles mais intelligibles. ${ }^{53}$ Pour Sydenham, cela revenait à présenter la vieille idée qu'il y a deux hommes en nous, l'homme visible et l'homme invisible, dans les termes de la physiologie moderne. Or ce que Boerhaave critique dans l'homme intérieur de Sydenham, permet de bien voir où lui-même a conscience d'innover: le médecin anglais a eu le tort, selon lui, de faire de l'homme intérieur et des maladies qui l'affectent, un homme et des maladies incorporelles. ${ }^{54}$ Pour Boerhaave (qui interprète mal la pensée de Sydenham), la dualité de l'homo duplex est celle de deux fonctions également corporelles, mais dont l'une est intérieure et dessine à elle seule une image du tout. L'homme double devient ici un homme doublement organisé, en deux fonctions hiérarchiquement situées l'une par rapport à l'autre, à l'intérieur d'une même corporéité.

L'idée d'un homme intérieur identifié avec son cerveau ou avec le système nerveux central, fera recette. Tout naturellement, c'est d'abord chez Gaub, le successeur de Boerhaave à Leyde, qu'elle réapparaît. Le De regimine mentis (1747) ne contient rien, selon moi, de très original, mais a le mérite d'insister sur la distinction des deux systèmes nerveux, volontaire et involontaire, tout en réservant la réponse à la question de leur «origine». Quoi qu'il en soit donc de ce problème, le cerveau et les nerfs qui en sont issus peuvent proprement être appelés un «homo nervosus»; si on les isolait du reste de l'organisme, ils dessineraient «une sorte de squelette, une image de l'homme». En ce sens, ils constituent bien «un homme à l'intérieur de l'homme». ${ }^{55}$ C'est probablement de Gaub, avec qui il était en relation, que Bonnet tirera sa définition du siège de l'âme: «Le siège de l'âme est une petite machine prodigieusement composée et pourtant fort simple dans sa composition. C'est un abrégé trés complet de tout le genre nerveux, une Neurologie en miniature». ${ }^{56}$ Et Cabanis, dans le troisième mémoire des Rapports $d u$ physique et du moral, évoquera à son tour le fameux passage de Sydenham, et fera du cerveau un «homme intérieur, doué des mêmes facultés, des mêmes affections, susceptible de toutes les déterminations analogues aux phénomènes extérieurs». ${ }^{57}$

Mais surtout, en 1753, Buffon intitule Homo duplex un long développement de son Discours sur la nature des animaux. "L'homme intérieur est double; il est composé de deux principes différents par leur nature et contraires par leur action" ${ }^{58}: l^{\prime}$ âme et le cerveau. L'homme est donc deux fois double: une première fois selon la dualité traditionnelle de l'homme intérieur 
identifié à son âme, et de l'homme extérieur, autrement dit le corps ${ }^{59}$. Mais cet homme extérieur est lui-même double, car il y a dans le corps deux parties: l'une qui agit sans interruption, et dont les foyers sont le cœur et les poumons; l'autre, sujette à des «pauses»; elle est constituée par les organes des sens et par les membres. La première est «intérieure»; la seconde est «l'enveloppe extérieure»: variable selon le degré de complexité des animaux, c'est elle qui fait leur spécificité. Cette «enveloppe» a, elle aussi, un intérieur, un centre: c'est le cerveau, que Buffon nomme «sens intérieur», ou «sens interne, général et commun». ${ }^{60}$ Telle est la seconde dualité de l'homme deux fois double: elle est dans la distinction et la correspondance du cerveau et des sens extérieurs: pas d'autre différence, entre eux, selon Buffon, que la généralité et la durée des «ébranlements» qui parviennent au cerveau, et la briéveté et la particularité des impressions faites sur les organes des sens extérieurs. «Pour tout le reste, [le cerveau] est de la même nature que les sens extérieurs. Le sens intérieur de l'animal est, aussi bien que ses sens extérieurs, un organe, un résultat de mécanique, un sens purement matériel.» ${ }^{61}$ Les sensations dont il est le siège sont de véritables «sensations corporelles» même si elles sont plus brèves que celles qui s'accomplissent dans l'âme, et que Buffon appelle «sensations de l'âme».

Quel destin auront ces analyses? Sur un point - la distinction de deux espèces de sensations -, Buffon subira les critiques convergentes du sensualisme et du cartésianisme. Condillac, au nom de «l'unité de l'être sentant», récusera dans le Traité des animaux (1755) l'idée qu'il y a en chacun de nous deux moi, deux personnes; une telle séparation rendrait tout commerce entre les deux parfaitement impossible, et, par voie de conséquence, tout conflit intérieur inintelligible ${ }^{62}$. Au nom d'une exigence philosophique très différente, Lelarge de Lignac fera à Buffon des reproches très semblables: qui, demande l'auteur des Lettres à un Américain (1756), a jamais fait l'expérience de ces «deux moi individuels qui se contredisent perpétuellement» ${ }^{63}$ ? Quel sens y a-t-il à dédoubler ainsi les sensations, et à mettre la douleur à la fois dans l'âme et dans le cerveau? A l'homo duplex de Buffon, Lignac n'oppose pas l'être sentant un cher à Condillac, mais l'homme de Descartes, composé d'un corps qui reçoit et transmet des «ébranlements», et d'une âme qui les perçoit.

Mais dans l'homo duplex de Buffon, il y a déjà, en un sens, Bichat ${ }^{64}$, et de nouveau Aristote. Bichat n'a pas pu ne pas voir en Buffon l'un de ceux qui ont «reconnu en nous deux espèces de principes», distingué «deux vies», l'une extérieure dont le cerveau est le centre, l'autre intérieure, dont le cœur 
et les poumons sont les «foyers» ${ }^{65}$. Comme Buffon, Bichat renverse l'ordre de dignité qui prévalait traditionnellement: l'homme essentiel, ce n'est pas l'homme intérieur, c'est au contraire l'homme extérieur, caractérisé par la vie de relation ${ }^{66}$. On sait qu'il l'appelle «vie animale», par opposition à une «vie organique». Or c'est pour décrire celle-là, que Bichat parle à son tour d'une vie qui «est pour ainsi dire double»: car il y a une «vie droite» et une «vie gauche», tandisque la vie organique est dissymétrique, irrégulière et confuse ${ }^{67}$. L'homme doit au fait de la bilatéralité une grande part de ses dons et de ses talents. Mais, en insistant sur la distinction des fonctions de nutrition et de relation, en montrant que la spécificité humaine réside dans la seconde et non dans la première, et aussi en faisant de la bilatéralité le propre de la vie animale, Bichat retrouve Aristote derrière Buffon: impossible en milieu aristotélicien où l'âme est «quelque chose du corps», ${ }^{68}$ la formule «l'homme est double» était remplacée dans les Parties des animaux par celleci: «le corps est double puisqu'il a une droite et une gauche». ${ }^{69}$ Galien commentera longuement cette assertion et beaucoup d'autres avec, ou après lui: la bilatéralité est l'une des conditions qui permettent la station droite, laquelle, à son tour, permet à l'homme de regarder vers les cieux. L'anthropologie chrétienne en fera l'un de ses thèmes de prédilection. Ainsi se trouvaient associées la dualité selon la droite et la gauche, et la dualité selon les parts mortelle et immortelle. D'ailleurs Boerhaave, dans son analyse de l'homo duplex, ne manque pas de rappeler cette signification: «en outre, cet homme corporel [constitué d'un homme extérieur et d'un homme intérieur également corporels] est ainsi formé qu'il est double, c'est-à-dire qu'il a une droite et une gauche». ${ }^{70}$ Aristote, Buffon, Bichat: il y a là une filiation que Bichat reconnaissait lui-même bien volontiers ${ }^{71}$.

Mais, dans l'homo duplex de Buffon, il y a aussi déjà Rousseau et Maine de Biran, et de nouveau Platon. Car la dualité de l'homme intérieur, partagé entre une âme et un cerveau est la source de tous les conflits: nous voulons ce que nous ne faisons pas et nous faisons ce que nous ne voulons pas: tel est le commentaire de Buffon qui ajoute: «Notre moi nous paraîtra ainsi divisé en deux personnes.» ${ }^{72}$ L'idéal ne serait-il pas alors dans une vie simple? Car, lorsque le sens intérieur matériel domine, ou lorsqu'au contraire c'est l'âme qui gouverne seule, «notre moi nous paraît simple parce que nous n'éprouvons qu'une impulsion simple: et c'est dans cette unité d'action que consiste notre bonheur». ${ }^{73}$ Les animaux, bornés au sens intérieur matériel, ont «une conscience d'existence simple»; «ils ne savent point qu'ils existent, mais ils le sentent». ${ }^{74}$ Comment ne pas tirer de ces considérations une apologie de la vie 
simple, transcription läque, exprimée dans le langage du sensualisme, d'une notion dont nous avons vu les antécédents platoniciens et chrétiens? Rousseau ne manquera pas de le faire: il lui suffira, comme l'a montré J.Starobinski, d'appliquer au sauvage ce que Buffon disait de l'animal. La «vie simple» qui précède l'éclosion de la civilisation aux dédoublements factices et aux masques trompeurs, nous préserverait aussi de la plupart des maladies. «L'homme n'est point un», s'écrie le Vicaire savoyard, qui ajoute comme on pouvait s'y attendre: «je vois le bien, je l'aime, et je fais le mal». ${ }^{75}$ Rousseau n'a rien à objecter, pour sa part, aux deux moi de Buffon ${ }^{76}$. D'où résulte une intense valorisation de la vie simple: n'est-ce pas elle que le promeneur solitaire connaît lorsqu'il jouit du «sentiment propre de l'existence», «dépouillé de toute autre affection» ${ }^{77}$ ? Or, parmi les lecteurs de cette admirable page, il y aura un jour le jeune Maine de Biran. Lui aussi connaît les déchirements de l'homo duplex et rêve d'une vie qui serait pure et simple fruition d'exister. Le 27 mai 1794, il est bien près d'en faire l'expérience, et il s'en souviendra toute sa vie ${ }^{78}$. Mais la simplicité sensitive n'est pas le propre de l'homme, et il faut revenir vite au redoublement douloureux de la réflexion. Il n'importe: le philosophe Maine de Biran saura édifier une philosophie originale sur cette duplicitas avec laquelle commence et finit l'humanité.

\section{Notes}

${ }^{1}$ Maine de Biran, Mémoire sur la décomposition de la pensée, éd. Tisserand, Paris, 1952, t. 1, p. $52-53$.

2 Boerhaave, Praelectiones academicae de morbis nervorum, Leyde, 1761, t.2, p. 497.

${ }^{3}$ Buffon, Histoire naturelle, De l'homme; je cite Buffon d'après l'édition des CEuvres complètes par le comte de Lacépède, Paris, Eymery, 1828, t. 10 et 11; cf. t.11, p. 399 sq.

4 Platon, République, III, 397 e; voir aussi VIII, $554 \mathrm{~d}$.

${ }_{5}^{5}$ Epictète, Manuel, XXIX, 7; voir aussi Platon, République, II, 370 c, III, 394 e, IV, $423 \mathrm{~d}$.

${ }^{6}$ Ecclésiastique, V, 9-10, Pour «cœur double», voir Osée, 10, 2; Psaumes, XII, 3; Ecclésiastique, I, 28 et $36, \mathrm{II}, 12$.

7 Jacques, I, 8 et IV, 8.

${ }^{8}$ Richelet, Dictionnaire de la langue française ancienne et moderne, Lyon, 1728, t.1. Richelet cite cette phrase de Montaigne: «Les hommes doubles sont utiles en ce qu'ils apportent; mais il faut se garder qu'ils n'emportent que le moins qu'on peut.» Amyot, traduisant les Vies parallèles de Plutarque emploie l'expression «homme double» (Alcibiade, L).

${ }^{9}$ Platon, République, IV, 439 b-e.

${ }^{10}$ Hippocrate, De natura hominis, 2, 3-4, éd. J. Jouanna, Berlin, 1975, p. 169.

112 Cor., 4, 16; Rom., 7, 22. 
${ }^{12}$ Entretien d'Origène avec Héraclide, éd. J.Schérer, Paris, 1960, p. 89 sq. Saint Augustin, De Genesi contra Manicheos, XVII, 27, PL 34, p.186.

${ }_{13}$ Poimandrès, 14, in Corpus Hermeticum, éd. Nock et Festugière, Paris, 1960, t. 1, p. 11.

${ }_{14}$ Asclepius, 7, ibid., t.2, p. 304.

${ }^{15}$ Grégoire de Nazianze, Oratio XXXVIII in Theophania, PG, 36, p.328 C. Voir aussi Eulogius d'Alexandrie, Sermo in ramos palmarum, PG 86, p. 2925 B.

${ }_{16}$ Nestorius, Sermo II, PL, 48, p. 764: «Sed idem ipse est duplex, non dignitate, sed natura.»

${ }^{17}$ Cyrille d'Alexandrie, Epistola 46 ad Succensum, PG, 77, p. 245 A.

${ }^{18}$ Cyrille de Jérusalem, Catechesis III illuminandorum, PG, 33, p. 430 A. Voir aussi Procope de Gaza, Commentarii in Genesim, PG, 87, p. $128 \mathrm{C}$.

${ }^{19}$ Grégoire de Nysse, De anima et resurrectione dialogus, PG, 46, p. 82 B. Jamblique, Traité de l'âme, in Festugière, La Révélation d'Hermès Trismégiste, Paris, 1953, t. 3, p. 192.

${ }^{20}$ Saint Bernard, Sermo XVII, PL, 183, p. 250: «Ibi vere vivitur, ubi vivida vita est et vitalis; De brevitate vitae, PL, 184, p. 1301: «Aeterna vita est vitalis, ista est mortalis.» Ruysbroeck, Le miroir du salut éternel, in Euvres, Bruxelles, 1912, t. 1, p. 134.

${ }^{21}$ Sur la diffusion des écrits hermétiques à la Renaissance, il faut lire le livre de F. Yates, Giordano Bruno and the Hermetic Tradition, Londres, 1964. Voir aussi E. Garin, Magie et Astrologie dans la culture de la Renaissance, in Moyen Age et Renaissance, trad. fr., Paris, 1968, p. 120-134.

${ }^{22}$ Pic de la Mirandole, De la dignité de l'homme, Lausanne, 1968, p. 24. Pour un commentaire de ce texte, voir entre autres P.O. Kristeller, Renaissance Thought, t.II, New York, 1965, p. 61 sq.

${ }^{23}$ Ibid., p. 32. C'est par l'expression «double nature» que Ficin avait traduit «duplex» dans son édition latine du Poimandrès. Cf. Ficin, Opera omnia, Bâle, 1576, t.2, p. 1837: «Solus homo ex universis terrenis animantibus duplicis naturae censetur.»

${ }^{24}$ Ficin, Opera omnia, t.I, p.473; Théologie platonicienne de l'immortalité de l'âme, trad. R. Marcel, Paris, 1970, t.3, p.123. Voir P.O. Kristeller, The Philosophy of Marsilio Ficino, New York, 1943, p. 197-198.

${ }^{25}$ Avicenne, Liber de anima, I, V. Sur le rôle des commentateurs latins dans la diffusion de l'image, cf. J. Rohmer «Sur la doctrine franciscaine des deux faces de l'âme», in Archives d'histoire doctrinale et littéraire du Moyen âge, 1927, et E. Gilson «Les sources gréco-arabes de l'augustinisme avicennisant», ibid., 1929-1930.

${ }^{26}$ Ficin, Théologie platonicienne..., t.3, p.123. Cf. E. Cassirer, Individu et cosmos dans la philosophie de la Renaissance, Paris, 1983, p.86sq.

${ }^{27}$ Voir notamment Jean Scot Erigène, Homélie sur le Prologue de Jean, XIX.

${ }^{28}$ Voir le livre important de D.P. Walker, Spiritual and Demonic Magic from Ficino to Campanella, Londres, 1958.

${ }^{29}$ Le Pimandre de Mercure Trismégiste, traduction et commentaire de François de Candale, Bordeaux, 1579, p.52. Voir J.Dagens, «Le commentaire du Pimandre de François de Candale», in Mélanges d'histoire littéraire offerts à Daniel Mornet, Paris, 1951, p.21-26.

${ }^{30}$ Poimandrès, op. cit., p.11.

${ }^{31}$ Lefèvre d'Etaples, qui fut le maître de Bovelles, édite en 1505 le Poimandrès avec un commentaire. Le De sapiente paraît en 1510. Nous suivons l'édition procurée par P. Magnard sous le titre Le livre du sage, Paris, 1982; elle est précédée d'un bel essai: «L'homme délivré de son ombre.»

${ }^{32}$ Le livre du sage, p. 89. 
33 Ibid., p. 215.

34 Ibid., p. 167.

${ }^{35}$ Cassirer, op. cit., p. 118.

${ }^{36}$ Cf. C. G. Nauert, Agrippa and the Crisis of Renaissance Thought, Urbana, 1965, p. 40.

37 Paracelse, Le livre Paragranum, in Euvres médicales, Paris, 1968, p. 54.

38 Ibid., p. 51 .

39 Ibid., p. 53 .

40 Ibid., p.57. Voir aussi Les Cinq livres des maladies invisibles et de leurs causes, ibid., p. 230-231; «On lui donne aussi le noble nom de microcosme, pour autant qu'il contient tous les phénomènes célestes, la nature terrestre, les propriétés aquatiques et les caractères aériens. Il contient la nature de tous les fruits de la terre, de tous les minerais de l'eau, toutes les constellations et les quatre vents du monde.»Voir A. Koyré, Paracelse, in Mystiques, spirituels et alchimistes du XVI $I^{e}$ siècle allemand, Paris 1971, p.85sq. Voir aussi W.Pagel, Paracelse. Introduction à la médecine philosophique de la Renaissance, s. 1, 1963.

${ }^{41}$ Op. cit., p. 191.

${ }^{42}$ S.W.I., X, p.643. Cf. aussi S.W.I, XII, p.10; I, IX, p.298. On trouvera une doctrine semblable chez Agrippa: De occulta philosophia, III, XXXVI.

${ }^{43}$ Cité par E. W. Kämmerer, Le problème du corps, de l'âme et de l'esprit chez Paracelse et chez quelques auteurs du XVII siècle, in Paracelse, Cahiers de l'Hermétisme, Paris, 1980, p. 135.

${ }^{44}$ Le livre Paragranum, op. cit., p. 47.

45 Ibid., p. 34.

${ }^{46}$ Grande Astronomie, in Paracelse, Cahiers de l'Hermétisme, p. 245.

${ }^{47}$ Bovelles, op. cit., p. 101. P. Magnard, en note, renvoie pour les antécédents de cette inversion à Origène, Grégoire de Nazianze et Ficin (p. 324).

${ }^{48}$ Boerhaave, Institutiones medicae (1713); trad. La Mettrie, Institutions de médecine, Paris, 1739, T.1, p. 13.

49 Boerhaave, Praelectiones de morbis nervorum, t.II, p.493. Sur les circonstances de cette publication, on consultera G.A.Lindeboom, Hermann Boerhaave. The Man and his Work, Londres, 1968. Sur les Praelectiones, cf. B.P.M.Schulte, "The concepts of Boerhaave on psychic function and psychopathology", in G.A. Lindeboom (ed.) Boerhaave and his time, Leyde, 1970, p. 93-101.

50 Ibid.

51 Ibid., p. 489.

52 Ibid., p.496-497.

${ }^{53}$ Sydenham, Dissertatio epistolaris ad Guillelmum Cole ... de affectione hysterica (1681), in Opera medica, Genève, 1749, p.261: «Quemadmodum etiam Homo quidam Exterior conspicitur ex partibus sensui obviis compaginatus, ita procul dubio et Interior est quidem Homo e debita spirituum serie et quasi fabrica constans, solo rationis lumine contemplandus».

${ }^{54}$ Boerhaave, Praelectiones, p. 497.

${ }^{55} \mathrm{Gaub}$, De regimine mentis, Leyde, 1747, p.41. Edition anglaise avec commentaire de L. J. Rather, Mind and Body in Eighteenth Century Medicine, Londres, 1965, p. 64.

${ }^{56}$ Bonnet, Essai de psychologie, in Euvres, t. VIII, p. 8.

${ }^{57}$ Cabanis, Rapports du physique et du moral de l'homme, éd. C. Lehec et J. Cazeneuve, Paris, 1956, t.I, p. 209.

${ }^{58}$ Buffon, Discours sur la nature des animaux, op. cit., t.11, p.399. CF. G.Barsanti, «Alle 
origini dell'antropologia, aspetti del dibattito sulla classificazione dell'uomo (1750-1840)», in Paradigmi, I, 1, 1983, p. 75 sq.

${ }^{59}$ De l'homme, ibid., t.10, p.15.

${ }^{60}$ Ibid., t.11, p. 356 .

${ }^{61}$ Ibid., p. 357.

${ }^{62}$ Condillac, Traité des animaux, I, II, éd. G. Le Roy, Paris, 1947, p. 342.

${ }^{63}$ Lelarge de Lignac, Suite des Lettres à un Américain, Hambourg, 1756, XVIII ${ }^{\mathrm{e}}$ Lettre, p. 3.

${ }^{64}$ Bichat, Recherches physiologiques sur la vie et la mort, I, VI, §4.

${ }^{65}$ Ibid., II, I.

${ }^{66}$ Ibid., I, VI, § I.

${ }^{67}$ Ibid., I, II, $\S \S$ I et II.

${ }^{68}$ Aristote, De l'âme, II, 2, 414 a.

${ }^{69}$ Aristote, Parties des animaux, II, 10,656 b.

${ }^{70}$ Bichat, Anatomie Générale, Considérations préliminaires, § VIII.

${ }^{71}$ Boerhaave, Praelectiones..., p. 497.

${ }^{72}$ Buffon, op. cit., t. 11, p. 401.

${ }^{73}$ Ibid., p. 402.

74 Ibid., p. 385.

${ }^{75}$ Rousseau, Emile, IV, O.C. IV, p. 583.

${ }^{76}$ Ibid., p. 584, en note.

${ }^{77}$ Cinquième Promenade, O.C., I, p. 1047.

${ }^{78}$ Voir Journal, éd. H. Gouhier, Neuchâtel, 1957, t. III, p. 3-9.

\section{Summary}

In 1805 the French philosopher Maine de Biran used the term "homo duplex" to describe two strata of human nature. This term is based on a concept from philosophical anthropology with a long tradition. In the article the concept of man's dual nature is analysed as it occurs in platonic and christian philosophy, in the works of renaissance thinkers and especially in the history of medicine. Maine de Biran's ideas are based mainly on those of Sydenham, Boerhaave, and Buffon. In his works "homo duplex" implies that elementary vital functions are dominated by the consciousness of the ego, in accordance with Boerhaave's phrase "homo duplex in humanitate, simplex in vitalitate".

François Azouvi

5 , rue St. Denis

F-29100 Boulogne 